\title{
Extreme Lateral Supracerebellar Infratentorial Approach to the Lateral Midbrain
}

\author{
M. Yashar S. Kalani ${ }^{1}$ William T. Couldwell ${ }^{2}$
}

Address for correspondence M. Yashar S. Kalani, MD, PhD, Department of Neurosurgery, University of Virginia, School of Medicine, PO Box 800212, Charlottesville, VA 22908, United States (e-mail: kalani@virginia.edu).

J Neurol Surg B 2018;79(suppl S5):S415-S417.

\begin{abstract}
This video illustrates the case of a 52-year-old man with a history of multiple bleeds from a lateral midbrain cerebral cavernous malformation, who presented with sudden-onset headache, gait instability, and left-sided motor and sensory disturbances. This lesion was eccentric to the right side and was located in the dorsolateral brainstem. Therefore, the lesion was approached via a right-sided extreme lateral supracerebellar infratentorial (exSCIT) craniotomy with monitoring of the cranial nerves. This video demonstrates the utility of the exSCIT for resection of dorsolateral brainstem lesions and how this approach

Keywords

- extreme lateral supracerebellar infratentorial

- mesencephalon

- brainstem

- surgery

- cavernous malformation gives the surgeon ready access to the supracerebellar space, and cerebellopontine angle cistern. The lateral mesencephalic safe entry zone can be accessed from this approach; it is identified by the intersection of branches of the superior cerebellar artery and the fourth cranial nerve with the vein of the lateral mesencephalic sulcus. The technique of piecemeal resection of the lesion from the brainstem is presented. Careful patient selection and respect for normal anatomy are of paramount importance in obtaining excellent outcomes in operations within or adjacent to the brainstem.

The link to the video can be found at: https://youtu.be/alw-O2Ryleg.
\end{abstract}

\section{Disclosure Statement}

The authors have no personal financial or institutional interest in any of the materials or devices described in this article.

Conflict of Interest

None.

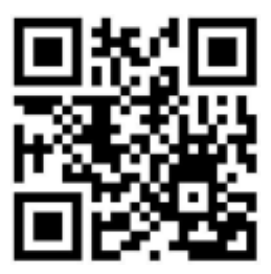

www.thieme.com/skullbasevideos

www.thieme.com/jnlsbvideos received

June 1, 2018

accepted

August 11, 2018

published online

September 25, 2018
DOI https://doi.org/

10.1055/s-0038-1669981. ISSN 2193-6331.
๑) 2018 Georg Thieme Verlag KG
Stuttgart · New York

License terms

() (i) $\ominus$ (\$) 

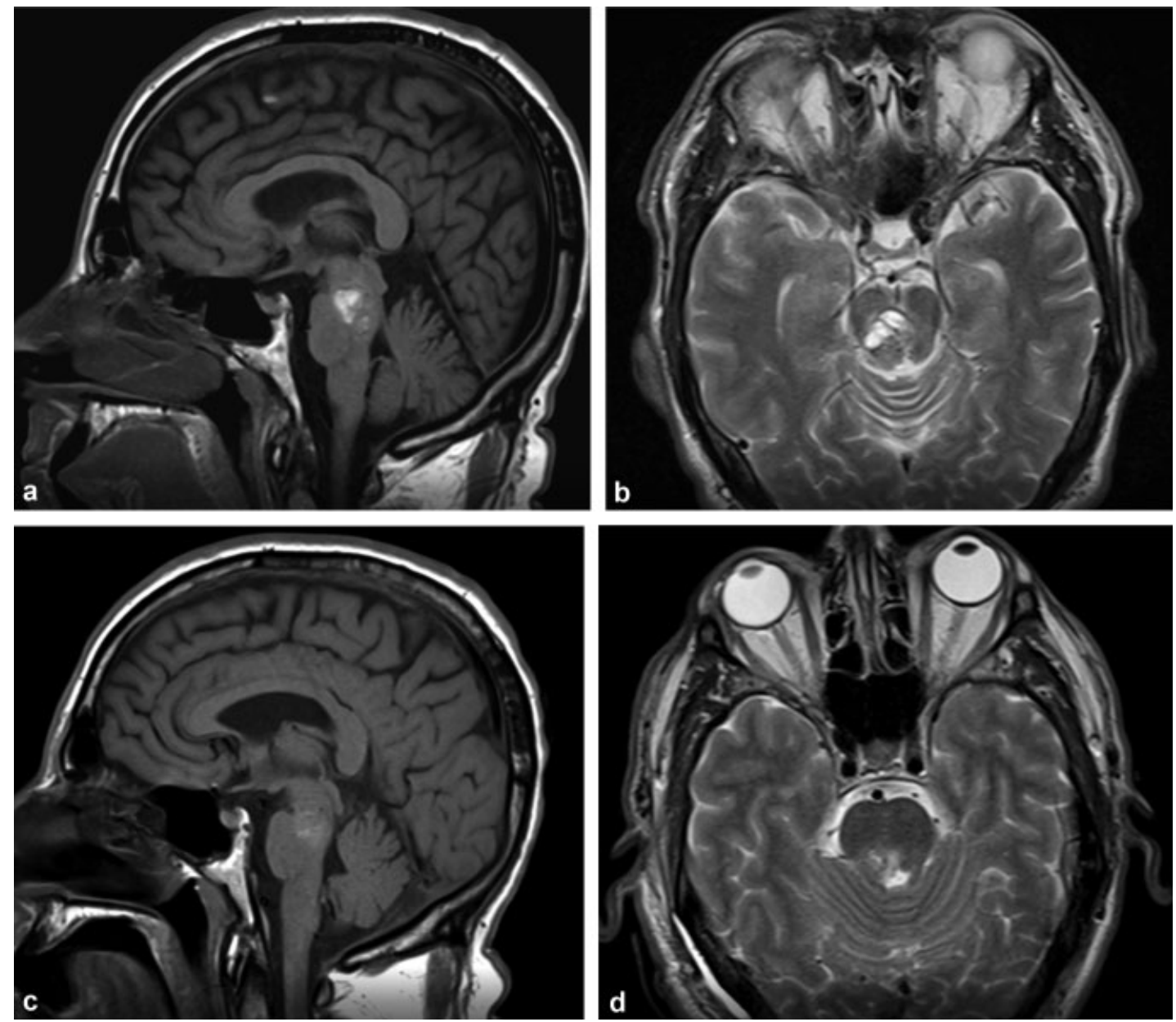

Fig. 1 (a) Sagittal T1- and (b) axial T2-weighted magnetic resonance imaging demonstrating a dorsolateral midbrain cavernous malformation with evidence of recent hemorrhage. Postoperative (c) sagittal T1- and (d) axial T2-weighted magnetic resonance imaging demonstrates complete removal of the lesion. 

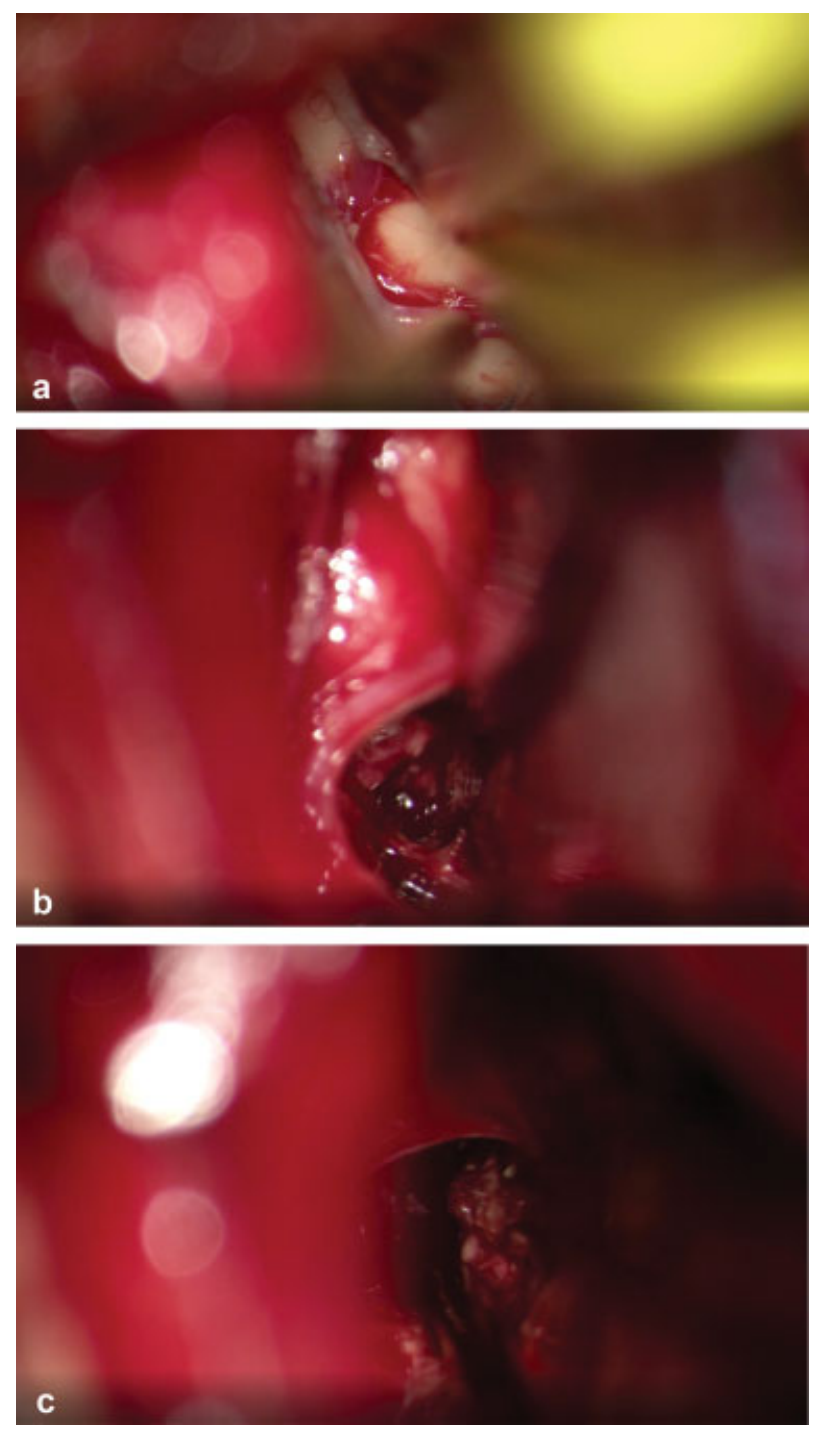

Fig. 2 Intraoperative photographs demonstrating (a) the lateral mesencephalic safe entry zone located at the intersection of the branches of the superior cerebellar artery and fourth nerve with the vein of the lateral mesencephalic sulcus, (b) mobilization of the cavernous malformation, and $(c)$ its removal from the brainstem. 